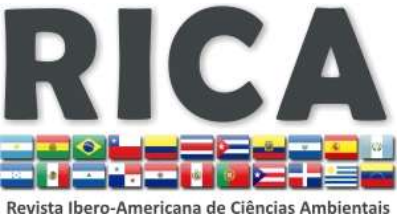

Journals Homepage:

www.sustenere.co/journals

\section{MODELO DE COBRANÇA PELO USO DA ÁGUA: ANÁLISE DA ARRECADAÇÃO NA SUBACIA DO ALTO PIRANHAS (PB)}

\section{RESUMO}

Por ser considerada um recurso essencial a vida, porém limitada, a água, possui potencial, por seus múltiplos usos, que exige necessidade de mecanismos que conduzam a uma utilização eficiente e racional deste bem. O objetivo principal deste trabalho foi estimar e analisar a arrecadação na sub-bacia do Alto Piranhas no Estado da Paraíba, por meio de um modelo de cobrança que considerasse as peculiaridades da região. Após levantamento dos principais modelos dispostos na literatura e por ser uma sub-bacia gerenciada pela esfera federal, observou-se que o modelo adotado pelo comitê da bacia do Rio São Francisco é o que mais se aproximou da realidade da região estudada. Assim foram idealizados e simulados dois cenários, a partir de demandas atuais e futuras que levassem em consideração o enquadramento dos corpos d' água. A partir disso notou-se que a arrecadação total anual do Cenário II é maior que o Cenário I, isso se deu principalmente com a mudança de alguns parâmetros que estão diretamente ligados as classes de enquadramento adotadas e dos níveis de garantias. Apesar de utilizando um modelo aprovado pelo Conselho Nacional de Recursos Hídricos (CNRH) e executado pela Agência Nacional de Água (ANA), os recursos gerados não foram suficientes para financiar o sistema de gerenciamento da bacia estudada. Dessa forma é preciso avaliar outros modelos de cobrança, assim como contemplar outros estudos que utilizem os coeficientes de ponderação que venham a considerar as peculiaridades da região estudada, visto que, este instrumento gera recursos capazes de possibilitar a melhoria da qualidade de vida da sub-bacia estudada.

PALAVRAS-CHAVES: Modelo de Cobrança; Arrecadação; Alto Piranhas.

\section{MODEL OF CHARGING FOR WATER USE: THE COLLECTION ANALYSIS IN SUBACIA THE HIGH PIRANHAS (PB)}

\section{ABSTRACT}

Since it is considered an essential life but limited water, has the potential for multiple uses resource to be need for mechanisms that lead to efficient and rational use of this asset. The main objective of this study was to estimate and analyze the storage in the sub-basin of the Upper Piranhas in Paraíba State, by means of a charging model that considered the peculiarities of the region. After a survey of the main models in the literature and willing to be a federal managed by sub-basin, it was observed that the model adopted by the São Francisco river basin committee is the one closest to the reality of the region studied. So were designed and simulated two scenarios, from current and future demands that took into account the classification of water bodies. From this it was noted that the total annual revenues of Scenario II is greater than Scenario I, it was mainly with changing some parameters that are directly linked classes adopted framework and levels of guarantees. Despite using a model approved by the National Water Resources Council (CNRH) and executed by the National Water Agency (ANA), the funds generated were not sufficient to finance the management system of the basin. Thus it is necessary to evaluate other charging models, as well as consider other studies that use the weightings that may consider the peculiarities of the region studied, since this instrument generates resources that can enable the improvement of quality of life sub- basin studied.

KEYWORDS: Chargingmodel; Collection; Alto Piranhas.
Revista Ibero-Americana de

Ciências Ambientais, Aquidabã, v.6, n.1, Dez 2014, Jan, Fev, Mar, Abr, Mai 2015.

ISSN 2179-6858

\section{SECTION: Articles} TOPIC: Recursos Hídricos

DOI: $10.6008 / S P C 2179-6858.2015 .001 .0019$

Francisca Rozângela Lopes de Sousa Universidade Federal de Campina Grande, Brasil http://lattes.cnpq.br/7564029475757969 rhozeadm@hotmail.com

Lilian Figueirôa Assis

Universidade Federal de Campina Grande, Brasil http://lattes.cnpq.br/3237162355201563

lilianfigueiroa@hotmail.com

Allan Sarmento Vieira

Universidade Federal de Campina Grande, Brasil http://lattes.cnpq.br/1584355117069605 allan.sarmento@ufcg.edu.br

Received: 24/01/2015

Approved: $14 / 10 / 2015$

Reviewed anonymously in the process of blind peer.

Referencing this:

SOUZA, F. R. L.; ASSIS, L, F.; VIEIRA, A. S.. Modelo de cobrança pelo uso da água: análise da arrecadação na subacia do Alto Piranhas (PB). Revista lbero-Americana de Ciências Ambientais, Aquidabã, v.6, n.1, p.246-261, 2015. DOI: $h$ ttp://dx.doi.org/10.6008/SPC2179 $\underline{658.2015 .001 .0019}$ 


\section{INTRODUÇÃO}

A água é um recurso natural essencial para manutenção da vida na terra, mas ao longo dos anos vem se tornando escassa em várias partes do mundo. Sabe-se que o consumo de água cresceu de forma exponencial devido principalmente ao crescimento populacional e a produção de alimentos (PNUD, 2006), o que se agrava, dada a escassez proveniente da poluição dos mananciais e degradação do meio ambiente. Essa condição exige valoração econômica deste bem, como forma de assegurá-lo para as futuras gerações. Para Tundisi (2006) quando a população aumenta, assim como os índices de desenvolvimento econômico, cresce também a necessidade de mais água, o que tem ocasionado mudanças no ciclo hidrológico para atender às demandas. A construção de reservatórios, com vasta exploração dos mananciais e aquíferos associados à contaminação das águas tem colocado em risco as redes de água em diversas regiões do planeta.

Segundo Forgiarini et al. (2007) na década de 90, no Brasil, foram aprovadas várias leis inovadoras, direcionadas às mudanças de paradigmas da gestão dos recursos hídricos. Tais alterações marcaram principalmente um novo tempo em que a descentralização na política de recursos hídricos, adotaria a bacia hidrográfica como unidade de gestão, quando a água passou a ser considerada como um recurso natural limitado e dotado de valor econômico. Nesse sentido, viu-se a necessidade de implementar o instrumento da cobrança pelo poder público, com a finalidade alavancar recursos para financiar o sistema de gerenciamento e as ações definidas nos planos das bacias hidrográficas (RAMOS, 2007).

Para Dias et al. (2010) a cobrança, é um instrumento, que não objetiva apenas, incrementar a arrecadação para o Estado, e sim garantir aos múltiplos usuários, através do financiamento de infraestrutura, o acesso a água, de forma eficiente e com a preservação dos mananciais. Destacando inclusive que a prática da cobrança pelo uso da água funciona também como um agente indutor da gestão participativa, descentralizada e integrada. Além disso, o pagamento por serviços de proteção a qualidade da água, que enfatizam a valoração econômica deste bem, são cada vez mais comuns nos países europeus e nos da América Latina (GROOT \& HERMANS, 2009; PAGIOLA et al., 2005).

Partindo do pressuposto que a cobrança é um instrumento que visa o uso racional, a proteção e a preservação da água, o presente trabalho abre a discussão sobre essa problemática e pretende investigar como está sendo feita a execução da cobrança pelo uso da água no Estado da Paraíba, especificamente na sub-bacia do Alto Piranhas, que é gerenciada pelo comitê de Bacia Piranhas-Açu. Para tanto, o objetivo principal deste trabalho é mensurar a arrecadação na bacia do Alto Piranhas no Sertão Paraibano, adotando um modelo de cobrança sustentável para região, com intuito de averiguar se os recursos gerados são suficientes para financiar o sistema de gerenciamento da bacia estudada.

\section{REVISÃO TEÓRICA}




\section{Entendendo o Problema}

Por ser considerado um recurso natural, essencial a vida, porém limitada, a água, possui um potencial, por seus múltiplos usos, exigindo, assim, a necessidade de estudar mecanismos que conduzam a uma utilização eficiente e racional deste bem. Essa necessidade é ressaltada por Padilla et al (2013) quando aponta que desde o final da década de sessenta há uma preocupação mundial com o meio ambiente, em especial com a problemática da água, que pode ser vislumbrada pelas iniciativas globais para resolver a crise ambiental.

Segundo o Relatório de Desenvolvimento humano (PNUD, 2006), cerca de 700 milhões de pessoas em 43 países vivem no limiar, quando o problema é o acesso à água doce. Essa dificuldade tem como fatores: o aumento da demanda por água de boa qualidade, o aumento populacional, expansão agrícola, da industrialização, poluição dos mananciais associado à má distribuição dos recursos hídricos no Brasil, todos esses fatores alertam para uma gestão de qualidade. Na região Nordeste, que conta com $29 \%$ da população e apenas $3,30 \%$ dos recursos hídricos nacionais, o Estado da Paraíba está em situação crítica, com ofertas hídricas de cerca de 1.440 e $1.320 \mathrm{~m}^{3}$ por habitante/ano (BRASIL, 2006).

A escassez qualitativa e quantitativa desse recurso gera sérios conflitos de uso, já que aumenta o consumo, o desperdício, e consequentemente a poluição acarretam uma série de problemas que até bem pouco tempo não recebiam a devida preocupação. Dias et al. (2010) lembra que a partir dos anos de 1980, começa a formar uma consciência sobre a necessidade de preservação dos recursos naturais. A Gestão dos Recursos Hídricos, avaliada como um instrumento, que orienta não só o poder público, mas também a sociedade, principalmente no que se refere à utilização e monitoramento dos recursos ambientais, naturais, socioculturais e econômicos, nesse caso na área de abrangência de uma sub-bacia hidrográfica, promove o desenvolvimento sustentável na região.

\section{A Política dos Recursos Hídricos}

Conforme Braga (2008) aponta no Brasil, as tentativas de gestão das águas iniciaram-se em 1934, com o Código das Águas, mas somente a partir de 1997, o modelo de gestão dos recursos hídricos foi reformulado a partir da Lei Federal n 9.433/97, onde está reconhece a água como um bem de domínio público e dotado de valor econômico, além disso, a gestão deve ser descentralizada, integrada e participativa, envolvendo usuários, sociedade civil e gestores. Com a promulgação da Lei a cobrança passa a ser vista sob dois aspectos que consiste no conceito de 'usuário pagador' e de "poluidor pagador", de forma para quem desperdiça e polui mais, paga mais. No Brasil, a mencionada legislação dispõe sobre cinco instrumentos: os planos de bacia, o enquadramento dos corpos d'água, a outorga dos direitos de uso da água, o sistema de informações sobre recursos hídricos, e a cobrança pelo uso da água. 


\section{O Instrumento da Cobrança}

Por ser a água um recurso natural imprescindível para vida terrestre, faz necessário que o uso desse bem, seja manejado de forma racional e justa. Uma forma de estabelecer esse controle é através dos instrumentos previstos na Política Nacional de Recursos Hídricos, que busca uma gerência com qualidade e eficiência, promovendo o bem-estar da sociedade dentro de um critério sustentável. Nesse tocante, a cobrança pelo uso da água, acredita-se, é um dos principais indutor na busca da eficiência alocação da água, encorajando, inclusive, a sua conservação, além de gerar recursos financeiros para ações nos planos de bacia, no que se refere a sua manutenção, preservação e recuperação. Porém, não há consenso entre os formuladores de políticas públicas e economistas de como deve ser conduzida a precificação da água, tendo em vista que não existem mercados para água ou estes não funcionam apropriadamente (TSUR, 2005).

Mas, a concordância de que a cobrança como instrumento é fundamental para equilibrar as necessidades e demandas, o que ocasiona consequentemente a conformidade entre usuários, ao passo que também aloca os custos sociais melhorando a qualidade dos efluentes lançados, além de arrecadar fundos financeiros para as obras, programas e intervenções do setor (MMA, 2005).

\section{MATERIAIS E MÉTODOS}

\section{Tipo de pesquisa}

O presente trabalho pode ser classificado como exploratório, descritivo, sem pesquisa de campo e de natureza quali-quantitativa. Isto porque, envolve pesquisa bibliográfica, estudo de caso e ainda estabelece relações entre variáveis. As observações foram estabelecidas de forma direta, porque se utilizou de instrumentos com finalidade de obter dados que evidenciaram o alcance dos objetivos propostos.

\section{Local da pesquisa}

A área de estudo compreende uma das sub-bacias do Rio Piranhas, abrangendo o seu alto curso, delimitada por dois reservatórios principais: o açude Engenheiros Ávidos e o açude de São Gonçalo. Os reservatórios de São Gonçalo e Engenheiro Ávido (figura 01) são obras hidráulicas construídas com o objetivo de reduzir os impactos advindos de problemas da escassez de água na região Nordeste do Brasil, e tem como características ser responsável pelo abastecimento humano e utilização pelo setor industrial e no Perímetro Irrigado de São Gonçalo, onde são desenvolvidas diversas culturas agrícolas, tanto permanentes, como coco, banana, capim, goiaba, acerola, manga, graviola e caju, quanto temporárias, como milho, feijão e batata. 


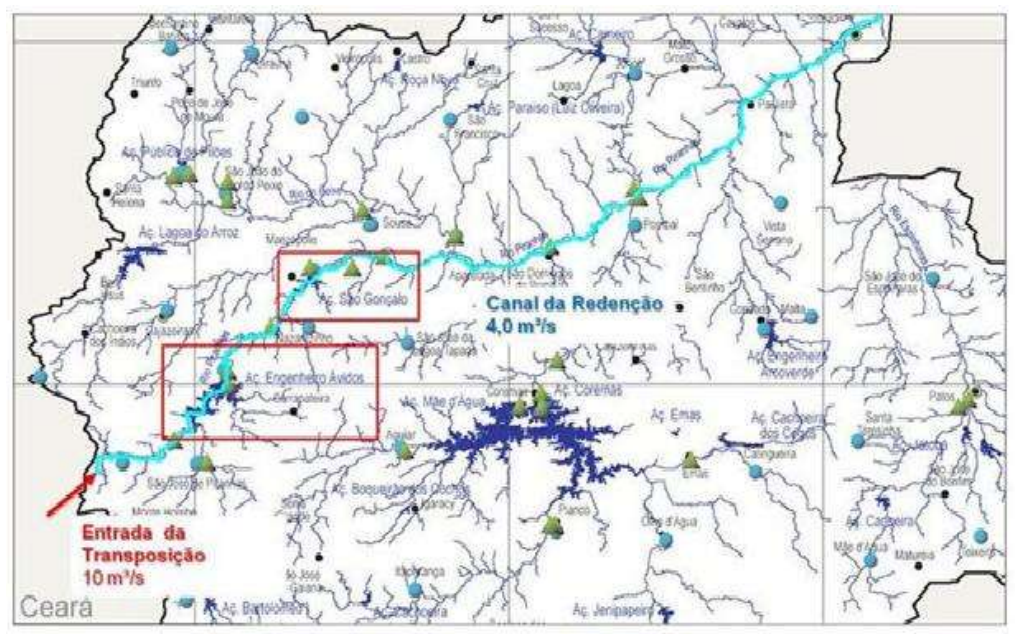

Figura 01: Subsistema de reservatórios na Bacia do Alto-Piranhas.

\section{Procedimentos}

Quanto ao desenvolvimento da pesquisa optou-se pelo método hipotético-dedutivo. Esta opção se justifica, porque o método escolhido permite o pesquisador propor uma hipótese e parte, por meio da dedução, para sua comprovação ou não. O material documentado e coletado, bem como, as respectivas análises dos resultados foi organizado na forma de um relatório final.

\section{Análise dos Dados}

Para colocar em ação os objetivos específicos propostos, foi feita inicialmente uma pesquisa bibliográfica e exploratória para entender os conceitos da cobrança pelo uso da água e levantar os principais modelos disponíveis para mensurar a arrecadação nas bacias hidrográficas do Brasil e do Estado da Paraíba.

Assim, inferiu-se a seguinte hipótese: 'Será que o modelo de cobrança a ser escolhido, que tem características financeiras, é adequado para sub-bacia do Alto Piranhas (PB) e ainda irá proporcionar uma arrecadação sustentável para execução das ações planejadas previstas pelo plano de bacia?'. Com o intuito de averiguar a veracidade da hipótese, optou inicialmente por uma coleta de dados da sub-bacia estudada a partir de banco de dados disponíveis em sites, do plano estadual de recursos hídricos e dos cadernos dos Comitês de Bacia Hidrográfica com atuação na área de influência.

Como a sub-bacia estudada está inserida numa gerência de âmbito federal, foi escolhido um modelo aprovado pelo Conselho Nacional de Recursos Hídricos (CNRH) e executado pela Agência Nacional de Água (ANA), dentre dos quais podemos citar, a metodologia aplicada na Bacia do Rio São Francisco. Assim a arrecadação foi estimada levando em consideração: a captação, consumo e lançamento de efluentes. Assim o valor a ser pago de cada usuário pela seguinte equação: 
Valortotal=(ValorCap+ValorCon+ValorLan+ValorTrans+ValorRu+ValorEle $)^{*}$ KGestã

o

(Eq. 1)

Onde:

Valortotal - pagamento anual da água referente a todos os usos;

ValorCap - pagamento anual referente à captação de água bruta feita pelos usos;

ValorCon - pagamento anual referente ao consumo de água bruta dos usuários;

ValorLan - pagamento anual referente ao lançamento de carga de DBO feitas pelo usuário;

ValorTrans - pagamento anual referente à transposição do rio São Francisco;

ValorRu - pagamento anual referente à captação e consumo no setor rural;

ValorEle - pagamento anual referente à produção de energia elétrica em pequenas usinas;

KGestão - Referente ao retorno efetivo dos recursos arrecadados, será considerado igual a 1 e quando não houver retorno a bacia será igual a zero.

A fórmula adequada para calcular o valor ser pago pela captação em cada usuário é:

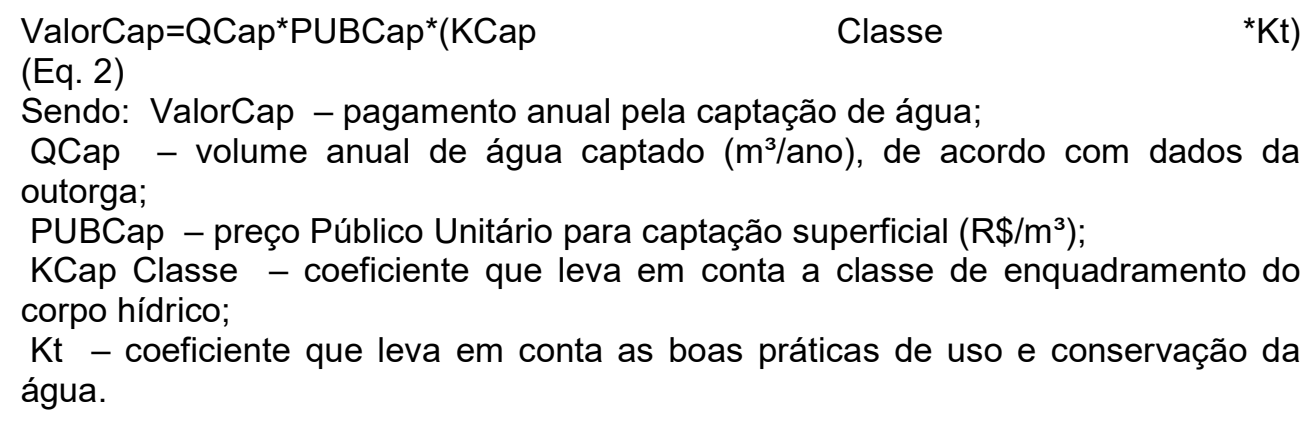

Os valores definidos para o coeficiente Kcap Classe as classes são apresentados no Quadro 01, variando entre 0,8 e 1,1.

Quadro 1: Valores aplicados na Bacia do Rio São Francisco.

\begin{tabular}{|l|l|}
\hline Classe de uso do corpo hídrico & \multicolumn{1}{c|}{ KCap Classe } \\
\hline 1 & 1,1 \\
\hline 2 & 1,0 \\
\hline 4 & 0,9 \\
\hline
\end{tabular}

Fonte: ANA, 2010.

Já na cobrança do valor pelo consumo, na bacia hidrográfica do Rio São Francisco é calculado pela equação a seguir:

\footnotetext{
ValorCon $=\left(\right.$ QCap-QLan) ${ }^{*}$ PUBCon ${ }^{*}$ KCon

(Eq. 3)

Sendo:

ValorCon - pagamento anual pelo consumo de água;

QCap - volume anual de água captado, segundo valores de outorga;

QLan - volume anual de água lançado, segundo valores de outorga;

PUBCon - preço Público Unitário para o consumo de água $\mathrm{R} \$ / \mathrm{m}^{3}$;

KCon - coeficiente que leva em conta os objetivos específicos a serem atingidos mediante a cobrança pelo consumo de água, é recomendado que seja igual a $\mathrm{K}_{\mathrm{t}}$, conforme deliberações do CBHSF.

No tocante ao valor a ser pago pelo uso dos recursos hídricos para diluição de carga poluente, é calculado pela fórmula a seguir:

ValorDBO $=(\mathrm{CDBO} * \mathrm{QLan})^{*} \mathrm{PUBLan}{ }^{*} \mathrm{KLan}$

(Eq. 4)

Onde:

ValorDBO - valor anual de cobrança pelo lançamento de carga orgânica (R\$/ano);

CDBO - concentração média anual referente à $\mathrm{DBO}_{5,20}$ do efluente lançado;

QLan - volume anual de água lançado;

PUBLan - preço Público Unitário para diluição de carga orgânica $(R \$ / \mathrm{kg})$;
} 
KLan - coeficiente que leva em conta os objetivos específicos a serem atingidos mediante a cobrança pelo lançamento de carga orgânica, que será igual a 1 (um) até nova deliberação do CBHSF.

A Nota Técnica $n^{\circ} 06$ da Agência Nacional de Águas (2010), menciona a questão da poluição, que necessita de uma determinada quantidade de água para diluir a sua carga poluente lançada no corpo hídrico, e é calculada por meio da multiplicação da concentração média anual referente à $\mathrm{DBO}_{5,20}$ do efluente lançado pelo volume anual de água lançado( $\left.\mathrm{Q}_{\mathrm{lan}}\right)$, como segue:

$$
\text { CODBO=CDBO } \times \text { QLan (Eq. 5) }
$$

Dessa forma os Preços Unitários Básicos (PUB) vigentes em 2010 e levados em consideração pelo Comitê de Bacia do Rio São Francisco ficaram estabelecidos nos seguintes valores. Com relação às projeções de demanda, tomou-se como referência o Plano Estadual de Recursos Hídricos da Paraíba (PERH), que mostra as demandas de abastecimento humano, pecuária, irrigação e industrial para os anos 2013, 2018 e 2023, anos que por sua vez, serviram de base para a projeção da cobrança, alvo desse estudo.

Quadro 2: Preço público unitário, segundo os usos da água.

\begin{tabular}{|l|l|l|l|}
\hline Tipo de uso & PUB & Unidade & Valor (R\$) \\
\hline Captação de água bruta & PUBcap & $\mathrm{R} \$ / \mathrm{m}^{3}$ & 0,01 \\
\hline Consumo de água bruta & $\mathrm{PUBcons}$ & $\mathrm{R} \$ / \mathrm{m}^{3}$ & 0,02 \\
\hline Lançamento de carga Orgânica $\mathbf{D B O}_{5,20}$ & $\mathrm{PUBDBO}$ & $\mathrm{R} \$ / \mathrm{m}^{3}$ & 0,07 \\
\hline
\end{tabular}

Fonte: (ANA, 2010).

Quadro 3: Demandas da Sub-bacia analisada ( $\left.\mathrm{m}^{3} / a n o\right)$.

\begin{tabular}{|l|l|l|l|l|}
\hline & Demandas (mªno) & $\mathbf{2 0 1 3}$ & $\mathbf{2 0 1 8}$ & $\mathbf{2 0 2 3}$ \\
\cline { 2 - 5 } Sub-bacia do Alto Piranhas & Humana Urbana & 7.477 .186 & 7.33 .489 & 7.448 .097 \\
\cline { 2 - 5 } & Humana Rural & 2.560 .364 & 2.479 .947 & 2.472 .183 \\
\cline { 2 - 6 } & Pecuária & 1.150 .614 & 1.150 .614 & 1.150 .614 \\
\cline { 2 - 6 } & Indústria & 69.495 & 74.458 & 80.064 \\
\cline { 2 - 6 } & Irrigação & 32.623 .026 & 39.009 .383 & 44.930 .325 \\
\cline { 2 - 5 } & Total & 43.880 .685 & 50.049 .251 & 56.081 .283 \\
\hline
\end{tabular}

Fonte: Adaptado - PERH-PB (2007).

Com a finalidade de obter melhor entendimento sobre essa arrecadação, foram idealizados e simulados os Cenários I e II, na qual considerou demandas passadas e futuras (2013, 2018 e 2023) estimadas no PERH-PB (BRASIL, 2006). Dessa forma, estes cenários possuem critérios de valores que podem ou não variar conforme os valores atribuídos, e dependem da componente (captação, consumo e lançamento), da classe de enquadramento (CONAMA, 357/05) e de outros elementos que dependem do modelo a ser escolhido. Assim no Cenário I adotou-se a Classe II e no Cenário II adotou-se a Classe III.

\section{Cenário I - Classe II}

Para o Cenário I, utilizou-se a Classe II, assim para a Componente Captação, o valor do

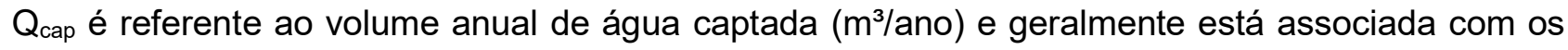
dados da outorga e o tipo de usuário. Devido à falta de dados consistentes, considerou-se como vazão de captação, as demandas atuais e futuras com perdas no percurso da água de $30 \%$ do 
volume estimado. Com relação ao $\mathrm{PUB}_{\text {cap }}$, optou-se por utilizar os mensurados pela ANA, com valores do ano de 2011, referente ao tipo de uso, que por sua vez foram atualizados levando em consideração a inflação média de $6 \%$ ao ano. $\mathrm{K}_{\text {classe }}$ é o coeficiente que leva em conta a classe de enquadramento do corpo hídrico, neste caso considerou-se a Classe II.

O Valor de Consumo, neste cenário, refere ao pagamento anual pelo consumo de água, ou seja, o valor medido. Foram considerados $70 \%$ do volume das demandas atuais e futuras, para os usos do abastecimento urbano e rural e a indústria, e $80 \%$ deste volume para os demais usos. Os PUB's foram atualizados considerando as perdas do sistema e a inflação média do ano. A

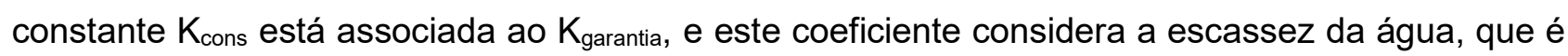
característica da região e são estabelecidos pela prioridade pelo tipo uso.

Tabela 1: Dados da Componente Captação.

\begin{tabular}{|c|c|c|c|c|c|}
\hline \multicolumn{6}{|c|}{ MODELO DE COBRANÇA DO VALOR DO COMPONENTE DE CAPTAÇÃO (Valorcap) } \\
\hline \multirow{3}{*}{ Ano } & \multirow{2}{*}{\multicolumn{5}{|c|}{$\begin{array}{l}\text { USOS MÚLTIPLOS DA ÁGUA } \\
\text { Abastecimento Humano (Urbano e Rural) }\end{array}$}} \\
\hline & & & & & \\
\hline & $Q_{\text {cap }}\left(\mathrm{m}^{3} / \mathrm{ano}\right)$ & PUB $_{\text {cap }}(\mathbf{R} \$)$ & $\mathrm{K}_{\text {classe }}$ & $\mathbf{K}_{\mathrm{t}}$ & Classe \\
\hline 2013 & 13.048 .815 & 0,0134832 & 1,0 & 1,0 & II \\
\hline 2018 & 12.759 .235 & 0,018043563 & 1,0 & 1,0 & II \\
\hline 2023 & 12.896 .364 & 0,024146358 & 1,0 & 1,0 & II \\
\hline \multirow{2}{*}{ Ano } & \multicolumn{5}{|l|}{ Pecuária } \\
\hline & $Q_{\text {cap }}\left(\mathrm{m}^{3} /\right.$ ano) & PUB $_{\text {cap }}(\mathrm{R} \$)$ & $\mathrm{K}_{\text {classe }}$ & $\mathrm{K}_{\mathrm{t}}$ & Classe \\
\hline 2013 & 1.150 .614 & 0,005618 & 1,0 & 1,0 & II \\
\hline 2018 & 1.150 .614 & 0,007518151 & 1,0 & 1,0 & II \\
\hline 2023 & 1.150 .614 & 0,010060982 & 1,0 & 1,0 & II \\
\hline \multirow{2}{*}{ Ano } & \multicolumn{5}{|l|}{ Indústria } \\
\hline & $Q_{\text {cap }}\left(m^{3} / a n o\right)$ & PUB $_{\text {cap }}(\mathrm{R} \$)$ & Kclasse & $\mathbf{K}_{\mathrm{t}}$ & Classe \\
\hline 2013 & 90.344 & 0,016854 & 1,0 & 1,0 & II \\
\hline 2018 & 96.795 & 0,022554454 & 1,0 & 1,0 & II \\
\hline 2023 & 104.083 & 0,030182947 & 1,0 & 1,0 & II \\
\hline \multirow{2}{*}{ Ano } & \multicolumn{5}{|l|}{ Irrigação } \\
\hline & $Q_{\text {cap }}\left(\mathrm{m}^{3} / \mathrm{ano}\right)$ & PUB $_{\text {cap }}(\mathbf{R} \$)$ & $\mathrm{K}_{\text {classe }}$ & $\mathbf{K}_{\mathrm{t}}$ & Classe \\
\hline 2013 & 32.623 .026 & 0,005618 & 1,0 & 1,0 & II \\
\hline 2018 & 39.009 .383 & 0,007518151 & 1,0 & 1,0 & II \\
\hline 2023 & 44.930 .325 & 0,010060982 & 1,0 & 1,0 & II \\
\hline
\end{tabular}

Tabela 2: Dados da Componente Consumo.

\begin{tabular}{|c|c|c|c|c|}
\hline \multicolumn{5}{|c|}{ MODELO DE COBRANÇA DO VALOR DO COMPONENTE CONSUMO (Valorcon) } \\
\hline \multirow{3}{*}{ Ano } & \multirow{2}{*}{\multicolumn{4}{|c|}{\begin{tabular}{|l|} 
USOS MÚLTIPLOS DA ÁGUA \\
Abastecimento Humano (Urbano e Rural) \\
\end{tabular}}} \\
\hline & & & & \\
\hline & $Q_{\text {con }}\left(m^{3} /\right.$ ano) & $\mathrm{PUB}_{\text {con }}(\mathrm{R} \$)$ & $\mathbf{K}_{\text {Garantia }}$ & Classe \\
\hline 2013 & 9.134 .171 & 0,00943824 & 0,99 & II \\
\hline 2018 & 8.931 .464 & 0,012630494 & 0,99 & II \\
\hline 2023 & 9.027 .455 & 0,01690245 & 0,99 & II \\
\hline \multirow{2}{*}{ Ano } & \multicolumn{4}{|l|}{ Pecuária } \\
\hline & $Q_{\text {con }}\left(m^{3} / a n o\right)$ & $\mathrm{PUB}_{\text {con }}(\mathrm{R} \$)$ & $\mathbf{K}_{\text {Garantia }}$ & Classe \\
\hline 2013 & 920.491 & 0,0044944 & 0,99 & II \\
\hline 2018 & 920.491 & 0,006014521 & 0,99 & II \\
\hline 2023 & 920.491 & 0,008048786 & 0,99 & II \\
\hline \multirow{2}{*}{ Ano } & \multicolumn{4}{|l|}{ Indústria } \\
\hline & $Q_{\text {con }}\left(m^{3} /\right.$ ano $)$ & $\mathrm{PUB}_{\text {con }}(\mathrm{R} \$)$ & KGarantia & Classe \\
\hline 2013 & 63.240 & 0,0117978 & 0,80 & II \\
\hline 2018 & 67.757 & 0,015788118 & 0,80 & II \\
\hline 2023 & 72.858 & 0,021128063 & 0,80 & II \\
\hline \multirow{2}{*}{ Ano } & \multicolumn{4}{|l|}{ Irrigação } \\
\hline & $Q_{\text {con }}\left(m^{3} /\right.$ ano $)$ & $\mathrm{PUB}_{\text {con }}(\mathrm{R} \$)$ & $\mathrm{K}_{\text {Garantia }}$ & Classe \\
\hline 2013 & 26.098 .421 & 0,0044944 & 0,90 & II \\
\hline 2018 & 31.207 .506 & 0,006014521 & 0,90 & II \\
\hline 2023 & 35.944 .260 & 0,008048786 & 0,90 & II \\
\hline
\end{tabular}

Tabela 3: Dados da Componente Lançamento Efluente.

\begin{tabular}{|c|c|c|c|c|}
\hline \multicolumn{5}{|c|}{ MODELO DE COBRANÇA DO VALOR DO COMPONENTE DE LANÇAMENTO (ValorLan) } \\
\hline \multirow{3}{*}{ Ano } & \multicolumn{4}{|c|}{ USOS MÚLTIPLOS DA ÁGUA } \\
\hline & \multicolumn{4}{|c|}{ Abastecimento Humano (Urbano e Rural) } \\
\hline & CODBO & PUB $_{\text {lan }}(\mathbf{R} \$)$ & $\mathrm{K}_{\mathrm{Lan}}$ & Classe \\
\hline 2013 & 3.196 .959 .675 & 0,01078656 & 1,0 & II \\
\hline 2018 & 3.126 .012 .526 & 0,01443485 & 1,0 & II \\
\hline 2023 & 3.159 .609 .180 & 0,019317086 & 1,0 & II \\
\hline \multirow{2}{*}{ Ano } & \multicolumn{4}{|l|}{ Pecuária } \\
\hline & CODBO & PUB $_{\operatorname{lan}}(\mathbf{R} \$)$ & $\mathrm{K}_{\text {Lan }}$ & Classe \\
\hline 2013 & 322.171 .920 & 0,0044944 & 1,0 & II \\
\hline 2018 & 322.171 .920 & 0,006014521 & 1,0 & II \\
\hline
\end{tabular}




\begin{tabular}{|c|c|c|c|c|}
\hline \multirow{3}{*}{$\begin{array}{l}2023 \\
\text { Ano }\end{array}$} & 322.171 .920 & $\mid 0,008048786$ & 1,0 & II \\
\hline & \multicolumn{4}{|l|}{ Indústria } \\
\hline & CODBO & $\mathrm{PUB}_{\operatorname{lan}}(\mathrm{R} \$)$ & $\mathrm{K}_{\text {Lan }}$ & Classe \\
\hline 2013 & 22.134 .158 & \begin{tabular}{|l|}
0,0134832 \\
\end{tabular} & 1,0 & II \\
\hline 2018 & 23.714 .873 & \begin{tabular}{|l|}
0,018043563 \\
\end{tabular} & 1,0 & II \\
\hline 2023 & 25.500 .384 & 0,024146358 & 1,0 & II \\
\hline \multirow{2}{*}{ Ano } & \multicolumn{4}{|l|}{ Irrigação } \\
\hline & CODBO & $\mathrm{PUB}_{\operatorname{lan}}(\mathrm{R} \$)$ & $\mathrm{K}_{\mathrm{Lan}}$ & Classe \\
\hline 2013 & 1.304 .921 .040 & 0,0044944 & 1,0 & II \\
\hline 2018 & 1.560 .375 .320 & \begin{tabular}{|l|}
0,006014521 \\
\end{tabular} & 1,0 & II \\
\hline 2023 & \begin{tabular}{|l|}
1.797 .213 .000 \\
\end{tabular} & \begin{tabular}{|l|}
0,008048786 \\
\end{tabular} & 1,0 & II \\
\hline
\end{tabular}

Para a análise da Componente Lançamento de Efluentes, a arrecadação será estimada com base na Demanda Bioquímica de Oxigênio $\left(\mathrm{DBO}_{5,20}\right)$ e está associada a quantidade de matéria orgânica contida no efluente, foi considerado uma média de $350 \mathrm{mg} / \mathrm{l}$ adotado pela Companhia de Saneamento Básico do Estado de São Paulo (SABESP). Já para a Irrigação, adotou-se a concentração média de $50 \mathrm{mg} / \mathrm{l}$. Quanto ao valor do PUB $\mathrm{B}_{\text {lan, }}$ adotou-se um coeficiente de retorno de 0,8, adotado pela CAGEPA (PB). É importante acrescentar neste cenário, informações relativamente importantes, de que a cobrança pelo lançamento de efluentes não deve ser vista como "licença para poluir", e sim modo de obrigar o poluidor a repor os gastos com o tratamento de efluentes, e que mesmo pagando pelo lançamento de efluentes, continua obrigado a atender os padrões de lançamento estabelecidos pelo órgão ambiental durante o licenciamento. (ANA, 2009).

\section{Cenário II - Classe III}

Em contrapartida, para o Cenário II, utilizou-se como base a Classe III, na Componente Captação, para este cenário a quantidade captada $Q_{\text {cap }}$ e $P U B_{\text {cap }}$ permaneceram os mesmos do cenário I, o que mudou foi o enquadramento que agora é a Classe III, o que consequentemente utilizou-se $\mathrm{K}_{\text {classe }}$ um valor de 0,9.

Tabela 4: Dados da Componente Captação.

\begin{tabular}{|c|c|c|c|c|c|}
\hline \multicolumn{6}{|c|}{ MODELO DE COBRANÇA DO VALOR DO COMPONENTE DE CAPTAÇÃO (Valorcap) } \\
\hline \multirow{3}{*}{ Ano } & \multicolumn{5}{|c|}{ USOS MÚLTIPLOS DA ÁGUA } \\
\hline & \multicolumn{5}{|c|}{ Abastecimento Humano (Urbano e Rural) } \\
\hline & $Q_{\text {cap }}\left(m^{3} / a n o\right)$ & PUB $_{\text {cap }}(\mathrm{R} \$)$ & $\mathbf{K}_{\text {classe }}$ & $\mathbf{K}_{\mathbf{t}}$ & Classe \\
\hline 2013 & 13.048 .815 & 0,0134832 & 0,9 & 1,0 & III \\
\hline 2018 & 12.759 .235 & 0,018043563 & 0,9 & 1,0 & III \\
\hline 2023 & 12.896 .364 & 0,024146358 & 0,9 & 1,0 & III \\
\hline \multirow{2}{*}{ Ano } & \multicolumn{5}{|l|}{ Pecuária } \\
\hline & $Q_{\text {cap }}\left(\mathbf{m}^{3} / \mathrm{ano}\right)$ & PUB $_{\text {cap }}(\mathbf{R} \$)$ & $\mathrm{K}_{\text {classe }}$ & $\mathbf{K}_{\mathbf{t}}$ & Classe \\
\hline 2013 & 1.150 .614 & 0,005618 & 0,9 & 1,0 & III \\
\hline 2018 & 1.150 .614 & 0,007518151 & 0,9 & 1,0 & III \\
\hline 2023 & 1.150 .614 & 0,010060982 & 0,9 & 1,0 & III \\
\hline \multirow[b]{2}{*}{ Ano } & \multicolumn{5}{|l|}{ Indústria } \\
\hline & $Q_{\text {cap }}\left(m^{3} / a n o\right)$ & PUB $_{\text {cap }}(\mathbf{R} \$)$ & $\mathbf{K}_{\text {classe }}$ & $\mathbf{K}_{\mathrm{t}}$ & Classe \\
\hline 2013 & 90.344 & 0,016854 & 0,9 & 1,0 & III \\
\hline 2018 & 96.795 & 0,022554454 & 0,9 & 1,0 & III \\
\hline 2023 & 104.083 & 0,030182947 & 0,9 & 1,0 & III \\
\hline \multirow{2}{*}{ Ano } & \multicolumn{5}{|l|}{ Irrigação } \\
\hline & $Q_{\text {cap }}\left(m^{3} / a n o\right)$ & PUB $_{\text {cap }}(\mathbf{R} \$)$ & $\mathbf{K}_{\text {classe }}$ & $\mathbf{K}_{\mathrm{t}}$ & Classe \\
\hline 2013 & 32.623 .026 & 0,005618 & 0,9 & 1,0 & III \\
\hline 2018 & 39.009 .383 & 0,007518151 & 0,9 & 1,0 & III \\
\hline 2023 & 44.930 .325 & 0,010060982 & 0,9 & 1,0 & III \\
\hline
\end{tabular}


Para a Componente Consumo, os valores de $\mathrm{Q}_{\text {con }}$ e $\mathrm{PUB}_{\text {con }}$ permanecem os mesmos quando comparados com o Cenário I, o que mudou foi os valores da garantia da água.

Tabela 5: Dados da Componente Consumo.

\begin{tabular}{|c|c|c|c|c|}
\hline \multicolumn{5}{|c|}{ MODELO DE COBRANÇA DO VALOR DO COMPONENTE CONSUMO (Valorcon) } \\
\hline \multirow{3}{*}{ Ano } & \multicolumn{4}{|c|}{ USOS MÚLTIPLOS DA ÁGUA } \\
\hline & \multicolumn{4}{|c|}{ Abastecimento Humano (Urbano e Rural) } \\
\hline & $Q_{\text {con }}\left(m^{3} /\right.$ ano $)$ & $\mathrm{PUB}_{\text {con }}(\mathrm{R} \$)$ & $\mathrm{K}_{\text {Garantia }}$ & Classe \\
\hline 2013 & 9.134 .171 & 0,00943824 & 0,99 & III \\
\hline 2018 & 8.931 .464 & 0,012630494 & 0,99 & III \\
\hline 2023 & 9.027 .455 & 0,01690245 & 0,99 & III \\
\hline \multirow{2}{*}{ Ano } & \multicolumn{4}{|l|}{ Pecuária } \\
\hline & $Q_{\text {con }}\left(\mathrm{m}^{3} /\right.$ ano $)$ & $\mathrm{PUB}_{\text {con }}(\mathrm{R} \$)$ & $\mathrm{K}_{\text {Garantia }}$ & Classe \\
\hline 2013 & 920.491 & 0,0044944 & 0,99 & III \\
\hline 2018 & 920.491 & 0,006014521 & 0,99 & III \\
\hline 2023 & 920.491 & 0,008048786 & 0,99 & III \\
\hline \multirow{2}{*}{ Ano } & \multicolumn{4}{|l|}{ Indústria } \\
\hline & $Q_{\text {con }}\left(m^{3} /\right.$ ano $)$ & PUB $_{\text {con }}(\mathbf{R} \$)$ & $\mathbf{K}_{\text {Garantia }}$ & Classe \\
\hline 2013 & 63.240 & 0,0117978 & 0,90 & III \\
\hline 2018 & 67.757 & 0,015788118 & 0,90 & III \\
\hline 2023 & 72.858 & 0,021128063 & 0,90 & III \\
\hline \multirow[b]{2}{*}{ Ano } & \multicolumn{4}{|l|}{ Irrigação } \\
\hline & $Q_{\text {con }}\left(m^{3} /\right.$ ano $)$ & $\mathrm{PUB}_{\text {con }}(\mathrm{R} \$)$ & $\mathrm{K}_{\text {Garantia }}$ & Classe \\
\hline 2013 & 26.098 .421 & 0,0044944 & 0,95 & III \\
\hline 2018 & 31.207 .506 & 0,006014521 & 0,95 & III \\
\hline 2023 & 35.944 .260 & 0,008048786 & 0,95 & III \\
\hline
\end{tabular}

$\mathrm{Na}$ Componente Lançamento de Efluentes, considerou-se os mesmos valores do Cenário I, com exceção o valor de $\mathrm{K}_{\mathrm{lan}}$.

Tabela 6: Dados da Componente Lançamento de Efluentes.

\begin{tabular}{|c|c|c|c|c|}
\hline \multicolumn{5}{|c|}{ MODELO DE COBRANÇA DO VALOR DO COMPONENTE DE LANÇAMENTO (ValorLan) } \\
\hline \multirow{3}{*}{ Ano } & \multicolumn{4}{|c|}{ USOS MÚLTIPLOS DA ÁGUA } \\
\hline & \multicolumn{4}{|c|}{ Abastecimento Humano (Urbano e Rural) } \\
\hline & CODBO & PUB $_{\text {cap }}(\mathrm{R} \$)$ & $\mathbf{K}_{\text {lan }}$ & Classe \\
\hline 2013 & 3.196 .959 .675 & 0,01078656 & 1,1 & III \\
\hline 2018 & 3.126 .012 .526 & 0,01443485 & 1,1 & III \\
\hline 2023 & 3.159 .609 .180 & 0,019317086 & 1,1 & III \\
\hline \multirow{2}{*}{ Ano } & \multicolumn{4}{|l|}{ Pecuária } \\
\hline & CODBO & $\mathrm{PUB}_{\text {cap }}(\mathrm{R} \$)$ & $\mathbf{K}_{\text {lan }}$ & Classe \\
\hline 2013 & 322.171 .920 & 0,0044944 & 1,1 & III \\
\hline 2018 & 322.171 .920 & 0,006014521 & 1,1 & III \\
\hline 2023 & 322.171 .920 & 0,008048786 & 1,1 & III \\
\hline \multirow[b]{2}{*}{ Ano } & \multicolumn{4}{|l|}{ Indústria } \\
\hline & CODBO & PUB $_{\text {cap }}(\mathrm{R} \$)$ & $\mathbf{K}_{\text {lan }}$ & Classe \\
\hline 2013 & 22.134 .158 & 0,0134832 & 1,1 & III \\
\hline 2018 & 23.714 .873 & 0,018043563 & 1,1 & III \\
\hline 2023 & 25.500 .384 & 0,024146358 & 1,1 & III \\
\hline \multirow{2}{*}{ Ano } & \multicolumn{4}{|l|}{ Irrigação } \\
\hline & CODBO & PUB $_{\text {cap }}(R \$)$ & $\mathbf{K}_{\text {lan }}$ & Classe \\
\hline 2013 & 1.304 .921 .040 & 0,0044944 & 1,1 & III \\
\hline 2018 & 1.560 .375 .320 & 0,006014521 & 1,1 & III \\
\hline 2023 & 1.797 .213 .000 & 0,008048786 & 1,1 & III \\
\hline
\end{tabular}

Após essa análise e com intuito de averiguar o retorno dos investimentos foi considerado o plano de investimentos (Tabela 19), para determinação dos custos na sub-bacia do Alto Piranhas, com base nas informações constantes no Resumo Executivo do Plano Estadual de Recursos Hídricos do Estado da Paraíba - PERH/PB (AESA, 2006), neste são considerados oito categorias de ações, sendo elas: Desenvolvimento Institucional; Planejamento e gestão; Conservação da quantidade e qualidade dos Recursos Hídricos; Gestão, recuperação e operação de açudes; Obras e serviços de Recursos Hídricos de interesse local; Obras e serviços de infraestrutura hídrica; Obras e serviços de saneamento; Conservação do solo e água e de ecossistemas. 
Respeitando o princípio da isonomia realizou-se o rateio desses investimentos no Estado por Bacia, sendo assim, esse rateio foi calculado a partir do percentual da área da sub-bacia do Alto Piranhas, que com 2.588,45 $\mathrm{km}^{2}$ corresponde a $4,6 \%$ da área total do Estado. A cobrança pela retirada da água bruta, assim como pelo lançamento de efluentes pode ser um instrumento eficaz na arrecadação desses investimentos. Como apontado por Macêdo (2006) esses dois modos de cobrança divide os programas para cada tipo de arrecadação, por exemplo, os programas diretamente relacionados à qualidade da água ou reuso e monitoramento está relacionado à arrecadação pela cobrança do lançamento de efluentes e por esse motivo devem ser apropriadas $100 \%$ para este tipo de cobrança (Tabela 19). Como a previsão para esses investimentos no PERH/PB considera um período de 20 anos e sendo necessário saber quanto desse total será para cada ano, o valor a ser arrecado pode ser determinado através de fluxo de caixa, amortizado para o período anual com taxa de juros de $12 \%$ ao ano (considerada pelo $\mathrm{PERH} / \mathrm{PB}$ a taxa interna de retorno mínima referente a análise custo benefício).

$\begin{array}{lc}\mathrm{PMT}=\mathrm{VP} & \mathrm{FVP}(12 \%, 20)=\underline{1-(1+0,12)^{-20}}=7,469443624 \\ (\mathrm{Eq} .6) & ; \\ \mathrm{FVP}(\mathrm{i}, \mathrm{n}) & 0,12\end{array}$

\section{RESULTADOS}

É importante ressaltar que a arrecadação estimada foi com base no modelo de cobrança idealizado para bacia do Rio São Francisco, já que a mesma possui características climáticas semelhantes. A análise seguiu ainda a estrutura idealizada por Cenários idealizados anteriormente e por componentes (captação, consumo e tratamento de efluentes).

\section{Cenário I : Modalidade Captação}

Os resultados obtidos na simulação deste Cenário estão apresentados nas tabelas a seguir. A componente de captação é constituída quando a cobrança pelo uso da água está de acordo com o volume efetivamente retirado. Assim, independente do usuário usar ou não a água, ele pagará pelo direito adquirido de poder usá-la, funcionando como um custo de disponibilidade. $\mathrm{Na}$ Tabela 7 apresenta a arrecadação anual na Sub-bacia estudada para os diferentes tipos de usuários. E observou-se que a irrigação é o setor que mais irá contribuir no financiamento do sistema ao longo dos anos.

Tabela 7: Arrecadação por Captação.

\begin{tabular}{|l|l|l|l|l|}
\hline Ano & Abastecimento & Pecuária & Indústria & Irrigação \\
\hline $\mathbf{2 0 1 3}$ & $\mathrm{R} \$ 175.939,78$ & $\mathrm{R} \$ 6.464,15$ & $\mathrm{R} \$ 1.522,65$ & $\mathrm{R} \$ 183.276,16$ \\
\hline $\mathbf{2 0 1 8}$ & $\mathrm{R} \$ 230.222,06$ & $\mathrm{R} \$ 8.650,49$ & $\mathrm{R} \$ 2.183,17$ & $\mathrm{R} \$ 293.278,44$ \\
\hline $\mathbf{2 0 2 3}$ & $\mathrm{R} \$ 311.400,22$ & $\mathrm{R} \$ 11.576,31$ & $\mathrm{R} \$ 3.141,54$ & $\mathrm{R} \$ 452.043,21$ \\
\hline
\end{tabular}

\section{Modalidade de Consumo}

Através desta modalidade, será cobrado apenas pelo volume de água bruta que efetivamente for consumida na Sub-bacia, reflete o valor econômico da água, estimulando-o a 
evitar desperdícios, pois cobra do usuário cada metro cúbico de água que de fato é necessário para consumir. A Tabela 8 mostra a arrecadação anual dos múltiplos usos quando for cobrado pelo consumo e observou-se que os setores que mais contribuem para sistema foram a irrigação e o abastecimento urbano e rural.

Tabela 8: Arrecadação por Consumo

\begin{tabular}{|l|l|l|l|l|}
\hline Ano & Abastecimento & Pecuária & Indústria & Irrigação \\
\hline $\mathbf{2 0 1 3}$ & $\mathrm{R} \$ 85.348,39$ & $\mathrm{R} \$ 4.095,69$ & $\mathrm{R} \$ 596,88$ & $\mathrm{R} \$ 105.567,07$ \\
\hline $\mathbf{2 0 1 8}$ & $\mathrm{R} \$ 111.680,72$ & $\mathrm{R} \$ 5.480,95$ & $\mathrm{R} \$ 855,80$ & $\mathrm{R} \$ 168.928,38$ \\
\hline $\mathbf{2 0 2 3}$ & $\mathrm{R} \$ 151.060,25$ & $\mathrm{R} \$ 7.334,75$ & $\mathrm{R} \$ 1.231,48$ & $\mathrm{R} \$ 260.376,89$ \\
\hline
\end{tabular}

\section{Modalidade Lançamento}

$\mathrm{Na}$ análise da componente lançamento de efluentes a cobrança foi feita levando em consideração a carga de matéria orgânica lançada no determinado manancial. A Tabela 9 mostra a arrecadação anual caso o gestor público venha investir nesta modalidade e observou-se ainda o quanto é caro tratar os efluentes gerados numa bacia hidrográfica. $\mathrm{O}$ abastecimento urbano e rural é setor que mais contribuem para financiar este tipo de ação.

Tabela 9: Arrecadação por Lançamento.

\begin{tabular}{|l|l|l|l|l|}
\hline Ano & Abastecimento & Pecuária & Indústria & Irrigação \\
\hline $\mathbf{2 0 1 3}$ & $\mathrm{R} \$ 34.484 .197,35$ & $\mathrm{R} \$ 1.447 .969,48$ & $\mathrm{R} \$ 298.439,27$ & $\mathrm{R} \$ 5.864 .837,12$ \\
\hline $\mathbf{2 0 1 8}$ & $\mathrm{R} \$ 45.123 .523,43$ & $\mathrm{R} \$ 1.937 .709,79$ & $\mathrm{R} \$ 427.900,81$ & $\mathrm{R} \$ 9.384 .910,19$ \\
\hline $\mathbf{2 0 2 3}$ & $\mathrm{R} \$ 61.034 .442,67$ & $\mathrm{R} \$ 2.593 .092,80$ & $\mathrm{R} \$ 615.741,39$ & $\mathrm{R} \$ 14.465 .382,63$ \\
\hline
\end{tabular}

\section{Cenário II: Modalidade Captação}

A diferença deste Cenário II com relação ao Cenário I consiste nas alterações da classe de enquadramento e dos níveis de garantias da água. Na Tabela 10 apresenta os valores arrecadados para a componente captação, quando a água bruta estiver enquadrada na Classe III. Essa mudança resultou numa diminuição nos valores da arrecadação se comparado ao Cenário I. Para o ano de 2023 houve uma queda na arrecadação anual de $10 \%$ para o uso do abastecimento urbano e rural.

Tabela 10: Arrecadação pela Captação.

\begin{tabular}{|l|l|l|l|l|}
\hline Ano & Abastecimento & Pecuária & Indústria & Irrigação \\
\hline $\mathbf{2 0 1 3}$ & $\mathrm{R} \$ 158.345,80$ & $\mathrm{R} \$ 5.817,73$ & $\mathrm{R} \$ 1.370,38$ & $\mathrm{R} \$ 164.948,54$ \\
\hline $\mathbf{2 0 1 8}$ & $\mathrm{R} \$ 207.199,85$ & $\mathrm{R} \$ 7.785,44$ & $\mathrm{R} \$ 1.964,85$ & $\mathrm{R} \$ 263.950,60$ \\
\hline $\mathbf{2 0 2 3}$ & $\mathrm{R} \$ 280.260,20$ & $\mathrm{R} \$ 10.418,68$ & $\mathrm{R} \$ 2.827,38$ & $\mathrm{R} \$ 406.838,89$ \\
\hline
\end{tabular}

\section{Modalidade Consumo}

A Tabela 11 mostra a arrecadação anual para componente consumo e quando os níveis de garantias forem alterados para os diferentes usos. Foram observados que as arrecadações anuais da indústria e da irrigação aumentaram quando comparados com o Cenário I. Isso se deu pelo o aumento da garantia da vazão disponível ao longo do tempo.

Tabela 11: Arrecadação pelo Consumo.

\begin{tabular}{|l|l|l|l|l|}
\hline Ano & Abastecimento & Pecuária & Indústria & Irrigação \\
\hline $\mathbf{2 0 1 3}$ & $\mathrm{R} \$ 85.348,39$ & $\mathrm{R} \$ 4.095,69$ & $\mathrm{R} \$ 671,49$ & $\mathrm{R} \$ 111.431,91$ \\
\hline
\end{tabular}




\section{Modalidade Lançamento}

Com relação à análise da Componente Lançamentos de Efluentes, considerou-se que a Demanda Bioquímica de Oxigênio (DBO5,20) permaneceria a mesma nos dois cenários, como também o PUBlan, a variação se deu no Klan de 1,0 para 1,1 que está associação a classe de enquadramento. Na Tabela 12, observa-se, que houve um aumento da arrecadação anual de $10 \%$, ocasionado pelo recebimento do efluente (manancial) ser enquadrado na classe III, requerendo uma maior eficiência na remoção da carga orgânica.

Tabela 12: Arrecadação pelo Lançamento de Efluentes.

\begin{tabular}{|l|l|l|l|l|}
\hline Ano & Abastecimento & Pecuária & Indústria & Irrigação \\
\hline $\mathbf{2 0 1 3}$ & $\mathrm{R} \$ 37.932 .617,09$ & $\mathrm{R} \$ 1.592 .766,42$ & $\mathrm{R} \$ 328.283,20$ & $\mathrm{R} \$ 6.451 .320,83$ \\
\hline $\mathbf{2 0 1 8}$ & $\mathrm{R} \$ 49.635 .875,77$ & $\mathrm{R} \$ 2.131 .480,77$ & $\mathrm{R} \$ 470.690,89$ & $\mathrm{R} \$ 10.323 .401,20$ \\
\hline $\mathbf{2 0 2 3}$ & $\mathrm{R} \$ 67.137 .886,93$ & $\mathrm{R} \$ 2.852 .402,08$ & $\mathrm{R} \$ 677.315,53$ & $\mathrm{R} \$ 15.911 .920,89$ \\
\hline
\end{tabular}

\section{Cenário I - Arrecadação Total}

Para o cenário I, a grande representatividade dos usos sobre o volume arrecadado devese ao volume cobrado pelo lançamento de volumes de água da Bacia Hidrográfica do Rio São Francisco, assim como os PUB's diferenciados, considerando a taxa média de inflação. Na Tabela 13 mostra a arrecadação anual total, caso a cobrança seja implementada na Sub-bacia estudada.

Tabela 13: Arrecadação Total

\begin{tabular}{|l|l|l|l|l|}
\hline Ano & Vcap (R\$) & Vcon (R\$) & Vlan (R\$) & ValorTotal (R\$) \\
\hline $\mathbf{2 0 1 3}$ & $367.202,74$ & $195.608,02$ & $42.095 .443,22$ & $42.658 .253,99$ \\
\hline $\mathbf{2 0 1 8}$ & $534.334,16$ & $286.945,86$ & $56.874 .044,21$ & $57.695 .324,23$ \\
\hline $\mathbf{2 0 2 3}$ & $778.161,27$ & $420.003,36$ & $78.708 .659,49$ & $79.906 .824,13$ \\
\hline
\end{tabular}

\section{Cenário II - Arrecadação Total}

Na Tabela 14 mostra que o Cenário II proporcionou uma a arrecadação média anual maior quando comparada com Cenário I. Isso se deu principalmente na mudança de alguns parâmetros como a classe de enquadramento do manancial e dos níveis de garantia.

Tabela 14: Arrecadação Total.

\begin{tabular}{|l|l|l|l|l|}
\hline Ano & Vcap (R\$) & Vcon (R\$) & Vlan (R\$) & ValorTotal (R\$) \\
\hline $\mathbf{2 0 1 3}$ & $330.482,47$ & $201.547,47$ & $46.304 .987,55$ & $46.837 .017,48$ \\
\hline $\mathbf{2 0 1 8}$ & $480.900,74$ & $296.437,74$ & $62.561 .448,64$ & $63.338 .787,12$ \\
\hline $\mathbf{2 0 2 3}$ & $700.345,14$ & $434.622,68$ & $86.579 .525,44$ & $87.714 .493,27$ \\
\hline
\end{tabular}

Quanto a análise dos investimentos, considera-se que até 2025 tal tipo de cobrança será responsável pelo financiamento de $R \$$ 8.202.927,00 para retirada de água bruta e $R \$$ 6.994.415,00 para lançamento de efluentes. Esses valores foram amortizados para o período anual, utilizando-se a equação do valor presente para juros compostos, com período de capitalização ( $n=20$ anos) e taxa de juros ( $i=12 \%)$, chegando aos seguintes valores:

$$
\begin{aligned}
& \text { Para retirada: } \\
& \mathrm{PMT}=\mathrm{VP} / \mathrm{FVP}=>\mathrm{PMT}=\mathrm{R} \$ 1.098 .197,859562558
\end{aligned}
$$


Para lançamento:

$\mathrm{PMT}=\mathrm{VP} / \mathrm{FVP}=>\mathrm{PMT}=\mathrm{R} \$ 936.403,7473321721$

Com base nos cenários (Tabela 16) o que se pode observar é que o valor arrecadado para retirada está abaixo do esperado, nos anos de 2013 e 2018, de custos em investimento na bacia, assim como quanto ao lançamento o valor arrecadado é superior ao que se tem previsto para aplicação na bacia conforme o PERH. Dessa forma é preciso avaliar outros modelos de cobrança, assim como contemplar outros estudos que utilizem os coeficientes de ponderação que venham a considerar as peculiaridades da região estudada, já que mesmo utilizando um modelo aprovado pelo Conselho Nacional de Recursos Hídricos $(\mathrm{CNRH})$ e executado pela Agência Nacional de Água (ANA), os recursos gerados não são suficientes para financiar o sistema de gerenciamento da bacia estudada.

Tabela 15 : Plano de investimento para a Bacia do Alto Piranhas, com base no PERH/PB (AESA, 2006).

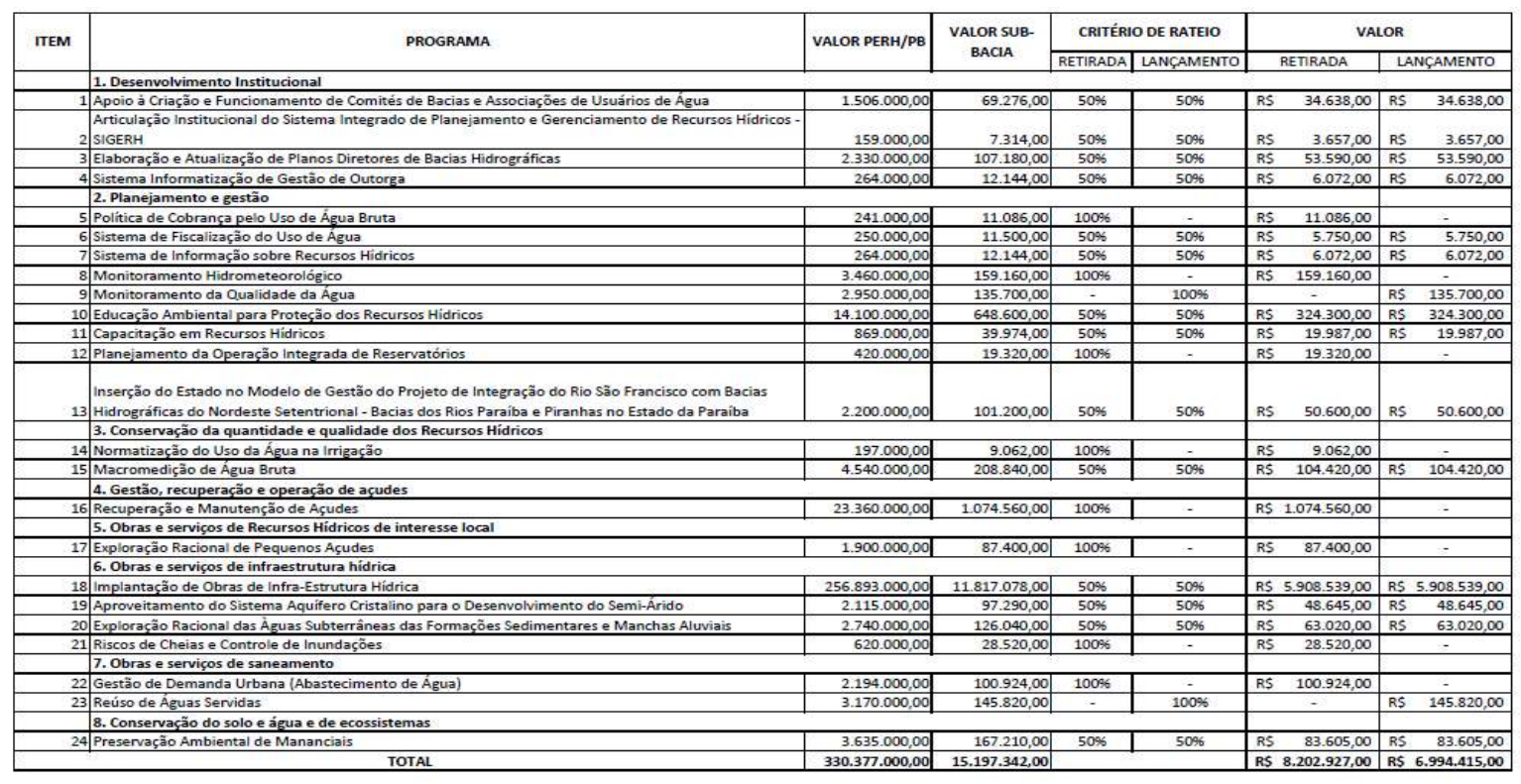

Tabela 16 : Arrecadação.

\begin{tabular}{|c|c|c|}
\hline \multicolumn{3}{|c|}{ CENÁRIO I } \\
\hline & Retirada (Captação+Consumo) & Lançamento \\
\hline 2013 & $562.810,76$ & $42.095 .443,22$ \\
\hline 2018 & $821.280,02$ & $56.874 .044,22$ \\
\hline 2013 & $1.198 .164,63$ & $78.708 .659,49$ \\
\hline \multicolumn{3}{|c|}{ CENÁRIO II } \\
\hline & Retirada (Captação+Consumo) & Lançamento \\
\hline 2013 & $532.029,93$ & $46.304 .987,54$ \\
\hline 2018 & $777.338,48$ & $62.561 .448,63$ \\
\hline 2013 & $1.134 .967,82$ & $86.579 .525,43$ \\
\hline
\end{tabular}

\section{CONCLUSÕES}

A importância de ampliar as pesquisas sobre os instrumentos da cobrança pelo uso água nas bacias hidrográficas, considerando os potenciais interesses dos usuários de diferentes setores da sociedade e a complexidade da bacia, é hoje o caminho para o fortalecimento da Política Nacional dos Recursos Hídricos. Assim para atender os objetivos desta pesquisa, inicialmente fez-se um levantamento dos principais modelos utilizados com a finalidade de estimar 
e conhecer a arrecadação na Sub-bacia do Alto Piranhas obedecendo as bases legais existentes que subsidiam implementação deste instrumento. Com base nas experiências existentes no Brasil e consideradas as peculiaridades da região resultaram na escolha de um modelo de cobrança aplicada na Bacia do Rio São Francisco.

Com base nos resultados observou-se que arrecadação total anual será diferenciada a depender da mudança dos parâmetros, como as classes de enquadramento dos corpos d água. Contudo este trabalho fundamentou-se no uso de um modelo cobrança que proporcionou o conhecimento da arrecadação anual das demandas atuais e futuras da Bacia do Alto Piranhas, alertando consequentemente o governo a urgência da implementação do instrumento da cobrança, tendo em vista que o que se deixa arrecadar poderia melhorar substancialmente a subbacia estudada. Além disso, viu-se a partir das estimativas dos custos das ações e do planejamento, que foram previstas pelo $\mathrm{PERH}$, que a arrecadação anual simulada não é suficiente para sustentar o sistema de gerenciamento da sub-bacia estudada, o que vem a sugerir a necessidade de estudos posteriores com a finalidade de construir um modelo pautado nas peculiaridades desta sub-bacia.

\section{REFERENCIAS}

BRAGA, B. P. F.; FLECHA, R.; PENA, D. S.; KELMAN, J.. Pacto federativo e gestão de águas. Estud. Av, v.22, n.63, p.17-42, 2008.

BRASIL. Agência Nacional de Águas (ANA). Implementação do enquadramento em bacias hidrográficas no Brasil. Sistema nacional de informações sobre recursos hídricos (SNIRH) no Brasil. Brasília: ANA, 2009.

BRASIL. Agência Nacional de Águas (ANA). Nota Técnica nº 06/2010/SAG. Cobrança pelo Uso de Recursos Hídricos na Bacia Hidrográfica do Rio São Francisco. Brasília: ANA, 2010.

BRASIL. Conselho Nacional de Meio Ambiente (COMANA). Resolução $\mathbf{n}^{\circ} .357$. Dispõe sobre a classificação dos corpos de água e diretrizes ambientais para o seu enquadramento, bem como estabelece as condições e padrões de lançamento de efluentes, e dá outras providências. Brasília: MMA, 2005.

BRASIL. Governo do Estado da Paraíba. Secretaria de Estado da Ciência e Tecnologia e do Meio Ambiente. Agência Executiva de Gestão de Águas do Estado da Paraíba, AESA. Brasília: Consórcio, 2006.

DIAS, T. F.; BARROS, H. O. M.; SOUZA, W. J.. Cobrança pelo uso da água: visões a partir dos membros do comitê de bacia hidrográfica do Rio Pirapama (PE). Revista Alcance, v.17, n. 4, p.416-432, 2010.

FORGIARINI, F. R., SILVEIRA, G. L.; CRUZ, J. C.. Cobrança pelo Uso da Água e Comitês de Bacia: Estudo de caso da Bacia Hidrográfica do Rio Santa Maria (RS). In: SIMPÓSIO BRASILEIRO DE RECURSOS HÍDRICOS. 17. Anais. São Paulo: ABRH, 2007.

GROOT, R. B. A.; HERMANS, L. M.. Broadening the picture: Negotiating payment schemes for water-related environmental services in the Netherlands. Ecological Economics, v.68, p.2760-2767, 2009.

PADILLA, J. H. R.; RINCÓN, M. A. P.; MALHEIROS, T. F.; PARRA, C. A. M.; PROTA, M. G.; SANTOS, R.. Análisis comparativo de modelos e intrumentos de gestión integrada del recurso hídrico en Suramérica: los casos de Brasil y Colombia. Revista Ambiente \& Água. An Interdisciplinary Journal of Applied Science, v.8, n.1, 2013.

PAGIOLA, S.; ARCENAS, A.; PLATAIS, G.. Can payments for environmental services help reduce poverty? An exploration of the issues and the evidence to date from Latin America. World Development, v.33, n.2, p. 237-253, 2005. 
PNUD. Água para lá da escassez: poder, pobreza e crise mundial da água. New York: Programa das Nações Unidas para o Desenvolvimento, 2006.

RAMOS, M.. Gestão de recursos hídricos e cobrança pelo uso da água. Rio de Janeiro: Escola Brasileira de Administração Pública, 2007.

TUNDISI, J. G.. Novas perspectivas para a gestão de recursos hídricos. Revista USP, São Paulo, n.70, p. 24-35, 2006.

TSUR, Y.. Economic aspects of irrigation water pricing. Canadian Water Resources Journal, v.30, n.1, p. 31-46, 2005.

VIEIRA, A. S.; SANTOS. V. S.; CURI. W. F.. Escolha das regras de operação racional para subsistema de reservatórios no Semiárido Nordestino. Revista Engenharia Ambiental, Espírito Santo do Pinhal, v.7, n.1, p.037-050, 2010. 